Anesthesia apparatus contamination \& prevention

\title{
An innovative approach to mitigating the risk of nosocomial infection during surgical anesthesia care
}

\section{Introduction}

There are significant infection control concerns in both provider behavior and equipment domains present in the surgical anesthesia workstation (AW), placing patients and their providers at substantial risk. ${ }^{1-5}$ There is a clinically significant potential for microbial cross-contamination from one patient to subsequent patients scheduled in the same operating room (OR) as a result of lapses in hand hygiene, procedural task density, equipment design complexity, and difficulty in disinfecting the apparatus between patients. (Figure 1)

During the anesthetic management of the surgical patient, the AW represents a challenging environment where it is nearly impossible to consistently perform optimal asepsis when rendering care. (Figure 2) Work from both our laboratory and clinical studies ${ }^{6-8}$, as well as from others $^{1-4,9}$, demonstrate quantifiable risks of microbial cross contamination in the AW that must be addressed. Health-care acquired infections (HAIs) are a significant national health concern carrying increased patient morbidity, mortality, and significant financial cost. ${ }^{10,11}$

Recent multidisciplinary expert guidance statements, endorsed by the American Operating Room Nursing Association, the American Society of Anesthesiology, the American Association of Nurse Anesthetists, and the American Association of Anesthesia Assistants, detail the grave risks of microbial iatrogenesis in the AW. ${ }^{5}$ Several of these recommendations relate to the need for ongoing patient safety research regarding between-case decontamination of the anesthesia delivery machine (ADM), as well as the potential role of disposable covers/wraps to prevent microbial contamination. This authoritative guidance paper noted a deficiency of, and need for, evidence-based research targeting these issues.

We designed a clinical pragmatic trial assessing a novel approach to mitigate the risk of cross contamination in the AW. We tested the hypothesis that strategic barriers placed on the anesthesia delivery machine during general anesthesia administration would decrease density and diversity of bacterial contamination over the course of sequential patients exposed to the same equipment, compared to machines without the barriers.

\section{Materials and Methods}

With IRB approval from Virginia Commonwealth University Medical Center (VCUMC), 30 diverse surgical cases requiring general anesthesia care over a 3-day period were matched 1:1 as the control group ( $\mathrm{N}=15$, no intervention, the uncovered condition) or the intervention group ( $\mathrm{N}=15$, condom-like barriers to anesthesia machine 'hot spots' defined as AW elements frequently touched and difficult to disinfect, revealed in our previous work, the covered condition). These included the electronic medical record (EMR) computer control mouse, the breathing circuit pressure control valve, the oxygen flowmeter control, and the anesthetic agent 
vaporizer dials.(Figure 3). This prospective, pragmatic trial involving a convenience sample of surgical patients was conducted in the main operating suites of VCUMC.

A supply of elasticized condom-like covers were obtained in three sizes (small, medium and large). In a pre-study test, we established that these were non-permeable to bacteria congruent with the full anesthesia machine wrap from our previous study. ${ }^{8}$

\section{Preliminary Study: assessing wrap integrity}

A preliminary study was performed to test the ability of the plastic covers to prevent bacterial contamination of the ADM. We applied a sterile condom-like cover to the vaporizer dial of an ADM. The dial was scrubbed clean with ethanol prior to the application to insure that prior contamination did not affect the results. Eswabs ${ }^{\mathrm{TM}}$, containing Aimes media, (COPAN Diagnostics Inc., Murrieta, CA) were then used to collect samples from the plastic cover (both sides) and the anesthesia machine prior to the inoculation with S. epidermidis (ATCC 12228). The sterile plastic cover was applied over the cleaned anesthesia machine dial. Approximately five colonies of S. epidermidis (ATCC 12228) were removed from a blood agar plate with a sterile swab and inoculated and spread on to the outside of the cleaned plastic cover. Eswabs ${ }^{\mathrm{TM}}$ were used to swab the outside of the plastic cover, inside of the plastic cover, and covered dial to determine if the plastic cover prevented the contamination of the ADM.

The collected swabs were taken back to the laboratory and vortexed for two minutes. Immediately following the vortexing, 100 microliters of Aimes media was removed from the $\mathrm{Eswab}^{\mathrm{TM}}$ tube and plated directly to a sheep blood agar plate (Thermo Scientific, Waltham, MA). The inoculum was spread evenly over the plate using a cell spreader, and the plates were incubated for 48 hours at $35^{\circ} \mathrm{C}$ to allow for bacterial growth. No bacterial growth was observed on the three plates prepared using samples collected prior to the addition bacteria indicating that the ADM (area covered) and plastic cover were free of detectable bacterial contamination prior to the inoculation with $S$. epidermidis. The only plate to demonstrate bacterial contamination from the samples collected after the addition of bacteria was the plate inoculated with the Eswab $^{\mathrm{TM}}$ from the outside of the plastic cover. Plates prepared using samples from the inside of the cover and the knob were both free of detectable bacterial contamination indicating that the covers prevents the contamination of the dial (Figure 4). The organism grown was then identified as $S$. epidermidis using Gram staining, catalase testing, coagulase testing, PYR test, and Novobiocin susceptibility testing.

\section{Conduct of the primary study}

\section{Ethical considerations}

With IRB ascent, the study was conducted in a manner that did not deviate from the routine surgical and anesthetic management of the enrolled patients. Enrollment of patients in the intervention group was approved by both surgical and anesthesia team members as well as the director of operating room services, all who were aware of the study goals and methodology. Confidentiality of patient information was assured as a given case became a randomly selected rubric and no identifying patient information of any kind was recorded. 
Culture samples were obtained from the four "hot spots" prior to the first surgical case of the day, after the provider had completed their routine anesthesia machine checkout procedure that ensures full functionality. This was done for each of the two matched rooms for each of the three days of the study. Rooms selected on each day of the study were matched based on case similarity with one room randomly chosen as the intervention (the covered condition) room, the other as the control (comparison, uncovered condition) room. To prevent contamination in the intervention room, the study team used hand sanitizer and donned gloves prior to placing the covers.

Immediately following each case, after both patient and provider exited the OR for transfer to the post anesthesia care area or ICU, repeated cultures were obtained from each of the "hot spots" after careful removal of the covering (employing the same hand sanitizing/gloving protocol as previously).

The researchers did not interfere with either the environmental services staff who routinely cleaned the AW between cases, nor the anesthesia technician staff who prepared the AW for the next case. The sequence was repeated in both the intervention (covered condition) OR and the control (uncovered condition) OR over the course of three consecutive days. (Figure 5)

\section{Bacterial Culture Analysis:}

Samples were collected for bacterial culture analysis using ESwabs ${ }^{\mathrm{TM}}$ (Copan Diagnostics Inc., Murrieta CA) from four designated target surfaces on the anesthesia machine in both the intervention and control rooms. Prior to use each Eswab ${ }^{\mathrm{TM}}$ was coated with tween, a nonionic surfactant, and excess solution was removed by pressing the tip of the swab against the wall of the tube. The target surface was swabbed using a rotating and twisting motion and immediately placed in one milliliter of liquid Aimes media. Once in the laboratory each Eswab ${ }^{\mathrm{TM}}$ was vortexed for two minutes and 100 microliters from each respective sample was removed to inoculate sheep blood agar (SBA) and MacConkey agar (Copan Diagnostics Inc., Murrieta CA). Inoculated agar plates were incubated at $35^{\circ} \mathrm{C}$ for 48 hours to observe bacterial growth. Colonies observed were examined based on colony morphology and colony forming units (CFUs) were recorded. Colonies were then sub-cultured on SBA for additional isolation and identification. Identification was based on colony morphology, Gram stain, and rapid spot tests such as catalase, coagulase, Staphaurex ${ }^{\mathrm{TM}}$ Plus latex agglutination, pyrrolidonyl arylamidase, Remel ${ }^{\mathrm{TM}}$ Microdase discs, indole, and oxidase (Thermo Fisher Scientific, Waltham MA).

\section{Post-procedural debriefing}

At the end of each case in the intervention group (covered condition) the 15 providers were given the opportunity to comment on the covers in a deliberately unstructured manner (to minimize bias) to asses any concerns or perceptions that they wanted to voice with regard to the covers.

\section{Data analysis}


Anesthesia apparatus contamination \& prevention

Mann-Whitney tests were used to examine the differences in total CFU density between the covered (intervention group) and uncovered (control group) conditions overall, between each case, between the four locations or "hot spots", and to establish baseline equivalency. MannWhitney is the non-parametric equivalent of the independent samples $t$-test and is appropriate to use when testing for differences across two independent conditions. Effect size $r$ was calculated and reported for each significant result. All data were analyzed using SPSS version 26.

\section{Results}

\section{Quantitative component: Bacterial contamination}

At the start of each day, baseline CFU density was equivalent in both conditions. Total CFU density was significantly lower in the covered, that is wrapped (Mean Rank $=5.81) v s$ uncovered, that is unwrapped condition (Mean Rank $=11.19$ ) at $p<0.01, r=-0.64$. This demonstrated a powerful protective effect of the wrapping material.

The ADMs from the intervention (covered) group demonstrated an overall decrease in colony forming units (CFUs) (Table 1). The highest CFU count for both groups was seen from samples collected from the oxygen flow valve. The control group had one case with $39,500 \mathrm{CFU} / \mathrm{mL}$ on this "hot spot" verses the intervention group which demonstrated a case with $660 \mathrm{CFU} / \mathrm{mL}$. Additionally, there were five cases $(5 / 16,31.25 \%)$ that demonstrated no bacterial contamination on any of the "hot spots" cultured in the intervention group. This was not the seen in the rooms that did not have covers over the "hot spots." All samples collected from these cases $(15 / 15)$ demonstrated bacterial contamination on at least one area tested.

Laboratory results demonstrated that these isolates were Gram-positive cocci in clusters, catalase positive, and coagulase positive. Isolate identification was confirmed to be $S$. aureus with a positive result using the Staphaurex ${ }^{\mathrm{TM}}$ Plus latex agglutination test.

There was a decrease in bacterial diversity for the covered oxygen flow valves, vaporizer dials, and computer mouse (Table 2). The oxygen flow knobs were the only "hot spot" contaminated with Staphylococcus aureus. This potential pathogen was isolated from two cases, both from the control group.

\section{Qualitative component: provider comments}

Post-case provider debriefings noted rare concerns regarding device performance that require follow-up modification. These primarily involved the device slipping off AW components (three of 15 providers), failed traction of the device on the associated component (two of 15 providers), and impeding smooth movement of the electronic medical record mouse (three ofn 15 providers). None of the concerns were considered insurmountable by the reporting provider and we are currently addressing the concerns with attention to fit and texture of device fabric.

\section{Discussion}


The Centers for Disease Control and Prevention published a white paper urging health care providers and institutional leadership to address the following short, and long-term goals: 1) preventing iatrogenic infection for those having surgical interventions, 2) preventing the spread of bacterial organisms, and 3) avoiding misuse of antibiotics. ${ }^{12}$ With this mandate, as well as that of the recent published guidelines ${ }^{5}$ urging research directed specifically at the AW, we undertook the current pragmatic trial.

The perioperative milieu is a reservoir of bacterial (and for that matter, viral and fungal) organisms that are pathogenic to humans. In recent work, it appears that $50 \%$ of surgical site infections are linked to bacterial pathogens that were in the OR where the patient's procedure was performed. ${ }^{4}$ There is compelling and methodologically sound research that demonstrates that the endemic nature of institutional health care acquired infection is due in part to the inherent complexity and strength of biofilm formation and its resistance to mechanical and chemical assault making aggressive action in hand hygiene, equipment cleaning/disinfection, and antibiotic stewardess critical areas to target. ${ }^{13,14}$

Anesthesia care routinely involves invasive procedures such as intravenous (IV) line placement, endotracheal intubation, and the use of needles to access nerves and the subarachnoid space, the latter to create what is known as regional anesthesia. These all bypasses the usual first-line defense mechanisms of the body. Open-lumen IV access stopcocks are used that are in direct and continuous continuity with the patient's circulating blood and are known to be contaminated with pathogenic organisms in about $1 / 3$ of surgical cases ${ }^{3,5,15}$ Using sophisticated techniques (e.g., cell genome identification, pulsed-field gel electrophoresis) organisms cultured at the stopcock lumen are directly linked to postoperative infection development as well as being associated with increased postoperative death. , $^{3,5,15}$

The presence of pathogens of any kind (bacterial, viral and fungal) on high touch areas (such as those we studied) and the ease of their transfer to a patient's intravenous access ports, and to their airway during even routine care, merits study and innovation to establish strategies that may prove beneficial in reducing the risk of AW-related infectious iatrogenesis. That providers wear disposable gloves is an essential component of patient care; 'gloving' or wrapping the hightouch, difficult to clean ADM components seems rational on a foundational level.

The few deficiencies and concerns noted in the qualitative component of the study were largely related to the wraps slipping of and are currently being addressed with attention to fit and texture of the device fabric. This occurred on two occasions with the $\mathrm{O}_{2}$ control, likely explaining contamination in the intervention group. There were no patient safety concerns raised (e.g., hindrance of care) by the providers in the intervention group. These deficiencies are currently being addressed with attention to fit and texture of device fabric.

With respect to disinfection of equipment prior to subsequent patients coming into contact with it, the covered condition (that is machines with wrap barriers in place) not only served as a barrier to contamination of apparatus 'hot spots' but served as a preventive measure to subsequent downstream (next-patient) exposure. This was a function of cover being removed at each case end thus mitigating the need for between-case disinfection. 
Anesthesia apparatus contamination \& prevention

While the study was conducted in a single, large metropolitan facility in the U.S., the implications are significant both domestically and internationally where infectious disease transmission remains a significant public health issue. The current coronavirus (COVID-19) crisis is but one condition that exemplifies the very real concerns with doing all that we can to mitigate the risk of pathogenic transmission in the health care setting.

\section{Limitations}

The study was not randomized and was performed at a single center. Although providers were not informed of the study purpose, and cultures were taken when they were not in the room, it is likely that a Hawthorne effect may have been present over the 3-day period. Furthermore, covers were not custom-designed to the ADM components, thus optimization of their effectiveness was not likely. Additionally, we only studied bacterial contamination; fungal (e.g., Candida auris) and viral (e.g., hepatitis C, COVID-19) pathogens were not assayed.

\section{Conclusion}

We demonstrated in previous work that use of a full apparatus barrier conveyed significant benefit to the patient in mitigating the risk of cross-contamination, yet may meet resistance by providers due to access constraints. In this proof of concept, clinical pragmatic trial, we demonstrated that strategic barrier applications to high-touch, universally contaminated niches on the ADM conveyed patient safety benefits by theoretically reducing the risk of AW-acquired infection, and add to the call for evidence noted in the recent authoritative guidance document. This research further validates the need for routine, periodic culturing of anesthetic apparatus to reveal lapses in provider behaviors and disinfection practices.

Future research should include a larger sample size, multi-institutional representation and an even broader patient population including obstetrical and pediatric patients. Likewise investigating the use of novel techniques such as that described in the current study targeting fungal and viral pathogens are warranted. 
Anesthesia apparatus contamination \& prevention

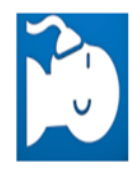

Patient

Oral secretions

Pulmonary secretions

Gastric contents

Mucosa

Skin

Blood

Foley

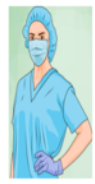

Anesthesia Provider

Hands

Behaviors

High task density

Multi-tasking

Confined space

Hand hygiene events very high

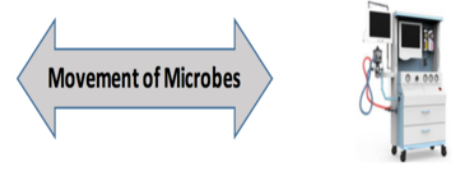

Anesthesia Machine/Equipment

Complex design

Hard to disinfect

Touched frequently/exposure common

Robust reservoir of pathogens

Figure 1: Vectors of microbial contamination in the anesthesia workstation 
Anesthesia apparatus contamination \& prevention

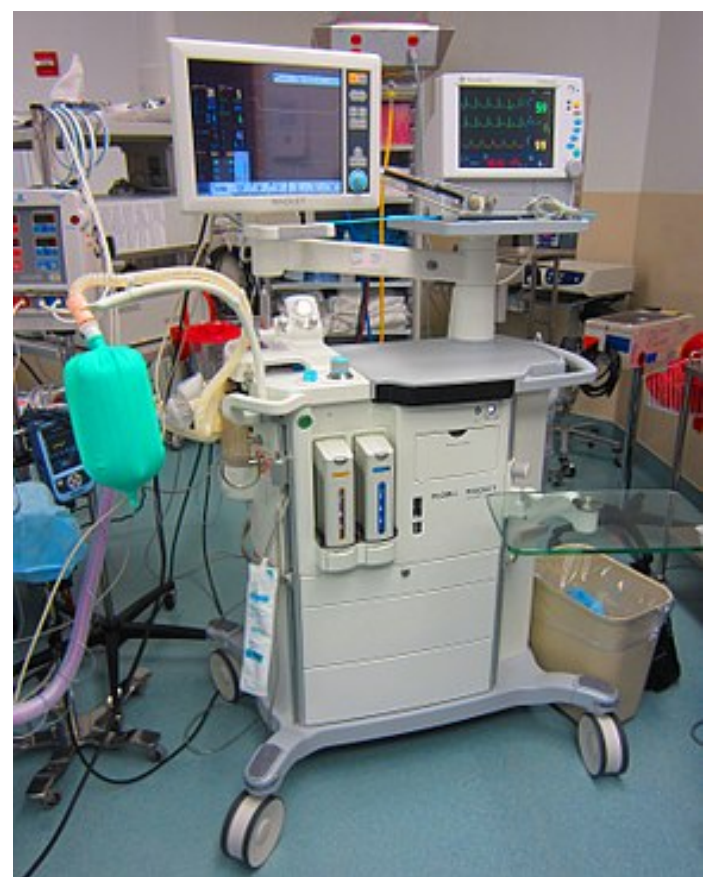

Figure 2: A typical anesthesia machine with architectural/design complexity making cleaning and disinfection between surgical cases very challenging. 


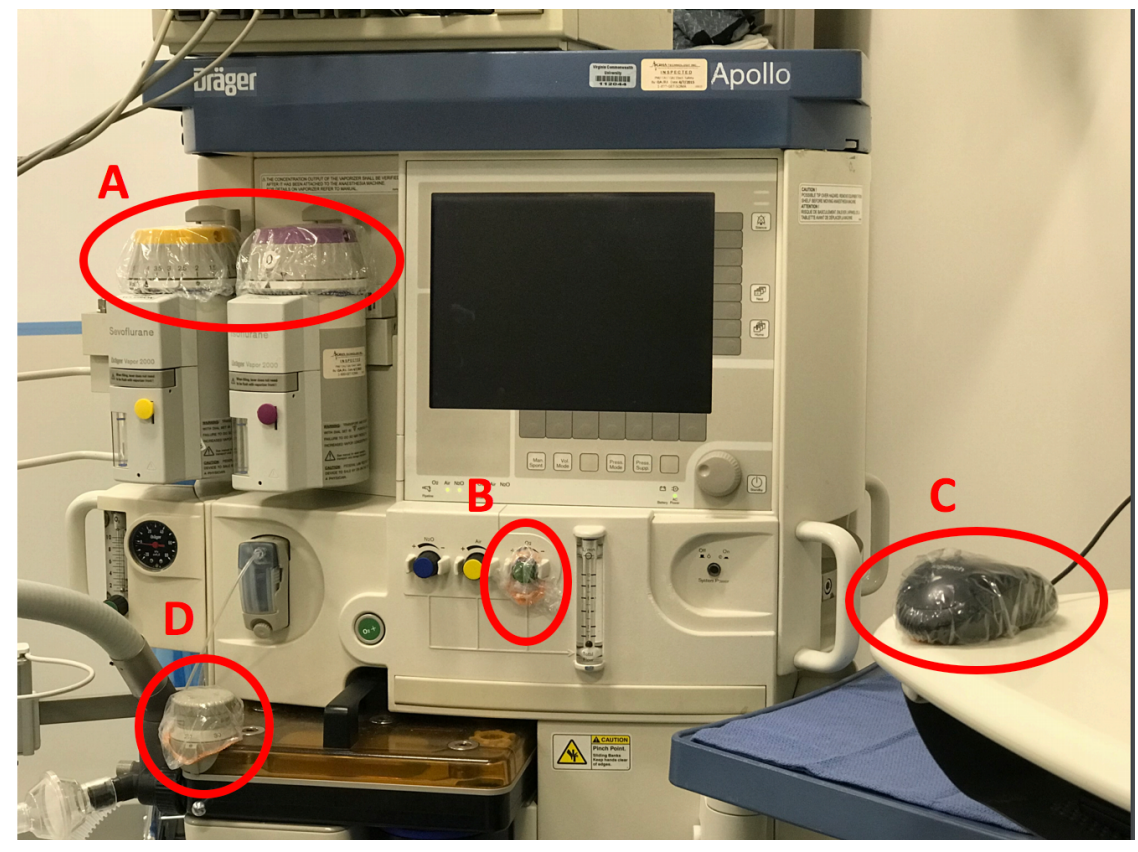

Figure 3: Location of condom-like protective covers

$\mathrm{A}=$ vaporizer control dials, $\mathrm{B}=\mathrm{O}_{2}$ flowmeter control, $\mathrm{C}=$ electrical medical record control mouse, $\mathrm{D}=$ breathing circuit pressure control valve (APL valve) 
Anesthesia apparatus contamination \& prevention
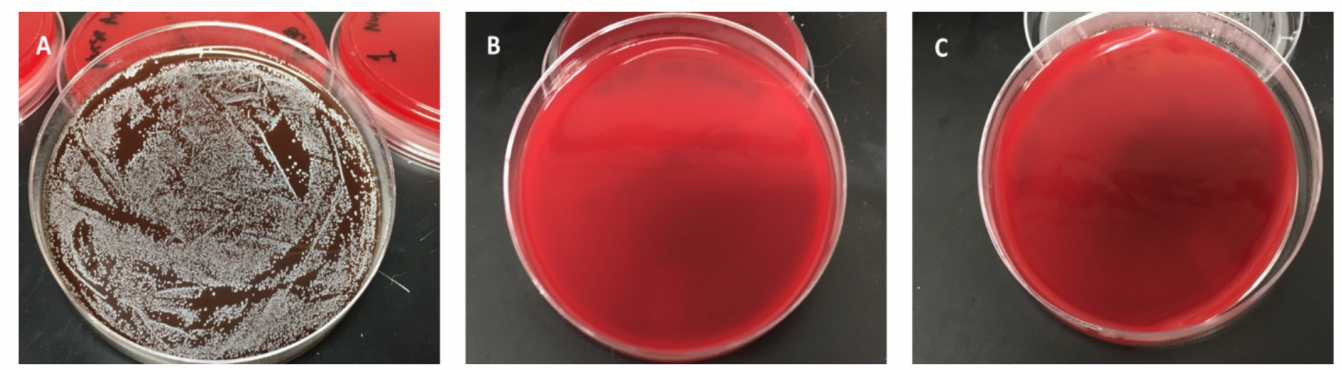

Figure 4: Preliminary study assess wrap barrier effectiveness

Growth on sheep blood agar after 48 hour incubation. A = outside of plastic cover after inoculation with $S$ epidermis. B = inside of plastic cover after inoculation with $S$ epidermis. $\mathrm{C}=$ knob covered after inoculation with $S$ epidermis. 
Anesthesia apparatus contamination \& prevention

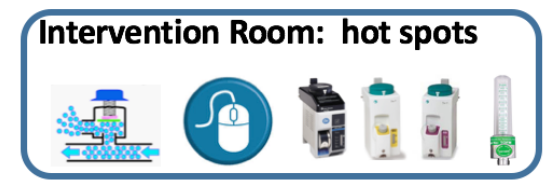

Baseline culturing
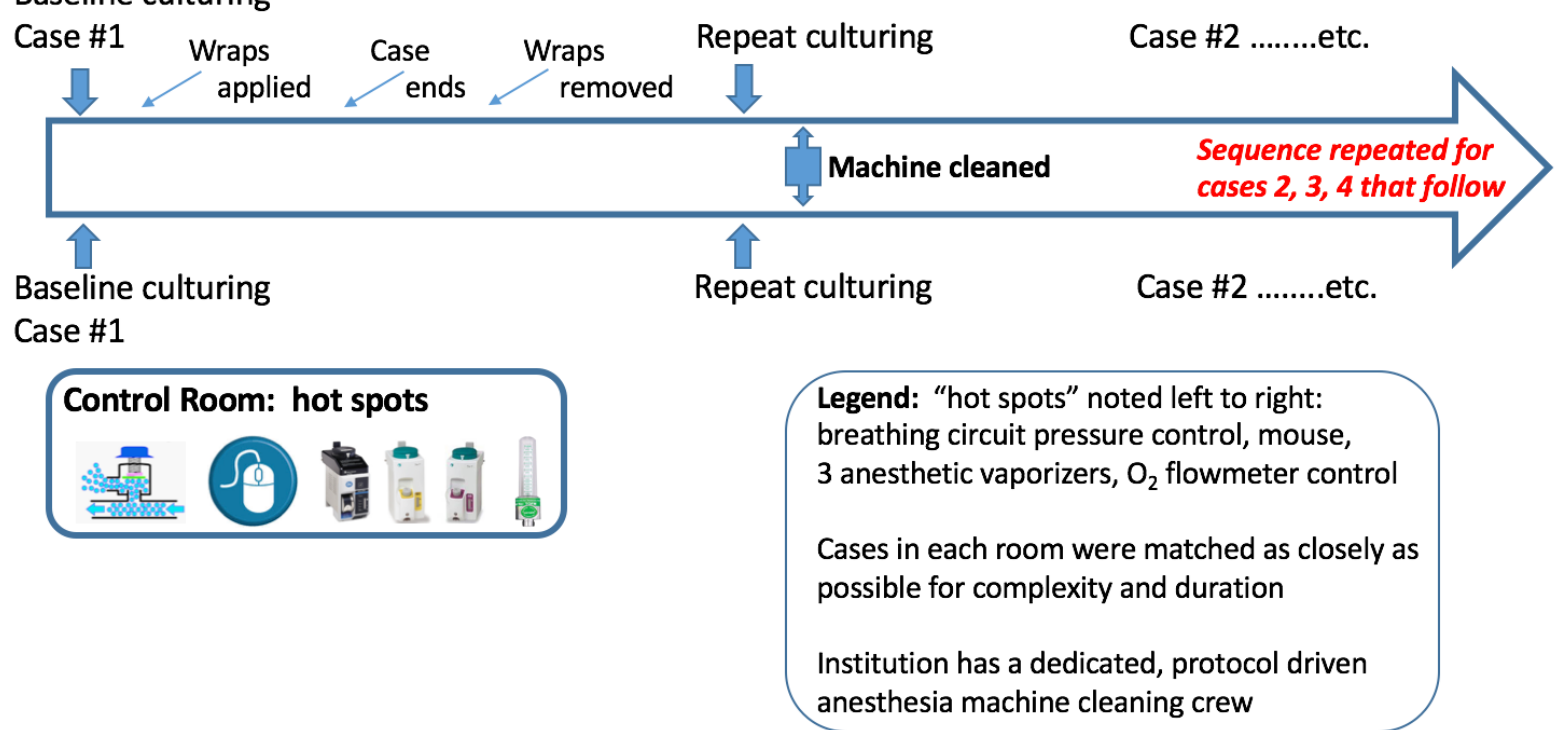

Figure 5: Chronology of culturing in the intervention \& control room cases 
Anesthesia apparatus contamination \& prevention

Table 1. Colony Forming Unit Range for Control Group vs Intervention Group

\begin{tabular}{|c|c|c|c|c|}
\hline Location & $\begin{array}{c}\text { Control Group } \\
\text { Colony Forming } \\
\text { Unit Range } \\
\text { (CFU/mL) }\end{array}$ & $\begin{array}{c}\text { Control Group } \\
\text { Median CFU } \\
\text { Count }\end{array}$ & $\begin{array}{c}\text { Intervention } \\
\text { Group } \\
\text { Colony Forming } \\
\text { Unit Range } \\
\text { (CFU/mL) }\end{array}$ & $\begin{array}{c}\text { Intervention } \\
\text { Group Median } \\
\text { CFU Count }\end{array}$ \\
\hline APL Valve & $0-1500$ & 8.50 & $0-90$ & $0.50^{* *}$ \\
\hline $\begin{array}{c}\text { Oxygen Flow } \\
\text { Valve }\end{array}$ & $0-39500$ & 4.50 & $0-660$ & $0.50^{* *}$ \\
\hline $\begin{array}{c}\text { Vaporizer } \\
\text { Dials }\end{array}$ & $0-240$ & 2.00 & $0-140$ & $0.00^{* *}$ \\
\hline $\begin{array}{c}\text { Computer } \\
\text { Mouse }\end{array}$ & $0-320$ & 10.00 & $0-80$ & $0.50^{* *}$ \\
\hline
\end{tabular}

$\mathrm{APL}=$ adjustable pressure limiting (breathing circuit pressure control valve)

** Significant difference $(\mathrm{p}<0.01)$ in $\mathrm{CFU} / \mathrm{mL}$ between the control (uncovered condition) and intervention (covered condition). 
Anesthesia apparatus contamination \& prevention

Table 2. List of bacteria cultured from the anesthesia machine

\begin{tabular}{|c|l|l|}
\hline Location & Control Group Organisms & Intervention Group Organisms \\
\hline APL Valve & $\begin{array}{l}\text { CoNS, Streptococcus } \text { species, } \\
\text { Micrococcus } \text { species }\end{array}$ & $\begin{array}{l}\text { CoNS, Bacillus } \text { species, } \\
\text { Streptococcus species, } \\
\text { Corynebacterium } \text { species, } \\
\text { Micrococcus } \text { species }\end{array}$ \\
\hline $\begin{array}{c}\text { Oxygen Flow } \\
\text { Knobs }\end{array}$ & $\begin{array}{l}\text { CoNS, Micrococcus } \text { species, } \\
\text { Streptococcus } \text { species, Bacillus } \\
\text { species, Staphylococcus } \text { aureus }\end{array}$ & $\begin{array}{l}\text { CoNS, } \text { Streptococcus } \text { species, } \\
\text { Bacillus } \text { species }\end{array}$ \\
\hline Vaporizer Dials & $\begin{array}{l}\text { CoNS, Bacillus } \text { species, } \\
\text { Streptococcus } \text { species }\end{array}$ & CoNS, Micrococcus species \\
\hline Computer Mouse & $\begin{array}{l}\text { CoNS, Micrococcus species, } \\
\text { Corynebacterium } \text { species, } \\
\text { Streptococcus } \text { species, Bacillus } \\
\text { species }\end{array}$ & $\begin{array}{l}\text { CoNS, Streptococcus } \text { species, } \\
\text { Bacillus } \text { species, } \text { Micrococcus }\end{array}$ \\
\hline
\end{tabular}

$\mathrm{APL}=$ adjustable pressure limiting; CoNS = coagulase-negative staphylococci 
Anesthesia apparatus contamination \& prevention

\section{References}

1. LS MunozPrice, DA Lubarsky, KL Arheart, G Prado, T Cleary, Y Fajardo-Aquino et al. Interactions between anesthesiologists and the environment while providing anesthesia care in the OR. Am J Infect Control, 2013;41:922-924.

2. Loftus R, Koff M, Burchman C, et al. Transmission of pathogenic bacterial organisms in the anesthesia work area. Anesthesiology. 2008;109:399-407.

3. Loftus RW, Brown JR, Koff MD et al. Multiple reservoirs contribute to intraoperative bacterial transmission. Anesth Analg. 2012;114:1236-1248.

4. Loftus RW, Dexter F, Robinson ADM. High-risk Staphylococcus aureus transmission in the operating room. Am J Infect Control. 2018;46:1134-1141.

5. Munoz-Price L, Bowdle A, Johnston L et al. Infection prevention in the OR anesthesia work area. Infect Control Hosp Epidem. 2019;40:1-17.

6. Biddle C, Shah J. Quantification of anesthesia providers' hand hygiene in a busy metropolitan OR: What would Semmelweis think? Amer J Infect Control. 2012;40:756759.

7. Biddle C, Robinson K, Pike B, Kammerman M, Gay B, Verhulst B. Quantifying the rambunctious journey of the anesthesia provider's hands during simulated care. Amer J Infect Control. 2016;44:873-878.

8. Biddle C, Gay B, Prasanna P, Hill E, Davis C, Verhulst B. Assessing a novel method to reduce anesthesia machine contamination: A prospective, observational trial. Can J Infect Dis Med Microbiol. 2018; Doi: 1905360.

9. Alysha D, Robinson B, Dexter F et al. Operating room PathTrac analysis of current intraoperative staphylococcus aureus transmission dynamics. Am J Infect Control. 2019;47:1240-1247.

10. Magill S, O'Leary E, Janelle S et al. Changes in prevalence of health care-associated infections in US hospitals. N Engl J Med. 2018;379;1732-1744.

11. The White House, Office of the Press Secretary. Fact Sheet: Actions to combat antibioticresistant bacteria. Available at: http://www.whitehouse.gov/the-pressoffice/2014/09/18/fact-sheet-obama-administration-takes-actions-combat-antibioticresistance.

12. Centers for Disease Control and Prevention. Healthcare associated infections. https://www.cdc.gov/hai/organisms/organisms.html. Accessed March 1, 2020.

13. Loftus RW, Dexter F, Robinson ADM, Horswill AR. Dessication tolerance is associated with Staphylococcus aureus hyper-transmissibility, resistance and infection development in the operating room. J Hosp Infect. 2018;100:299-308.

14. Allegranzi B, Kilpatrick C, Storr J et al. Global infection and control priorities 2018-22: a call for action. The Lancet. 2017;5:e1178-e1180.

15. Hadder B, Patel HM, Loftus RW et al. Dynamics of intraoperative Klebsiella, Acinetobacter, Pseudomonas and Enterobacter transmission. Am J Infect Control. 2018;46:107:A1788. 


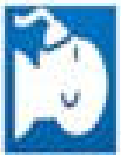

\section{Patient}

Oral secretions

Pulmonary secretions

Gastric cortents

Mucosa

Skin

Bood

Foley

\section{Anesthesia Provider}

Hands

Beharions

High task descity

Mutituasing

Confned space

Hand hygene events very high

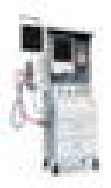

Anesthesia Machine/Equipment

Complex desizn Hard to disinfect

Touched frequent/Wexposure common Robust reservoir of pathogens 


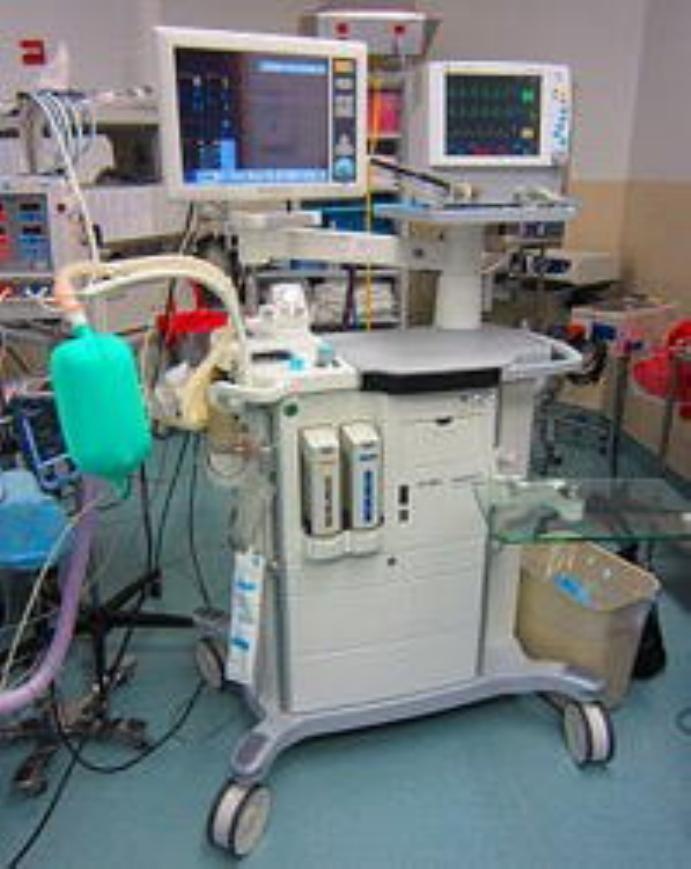




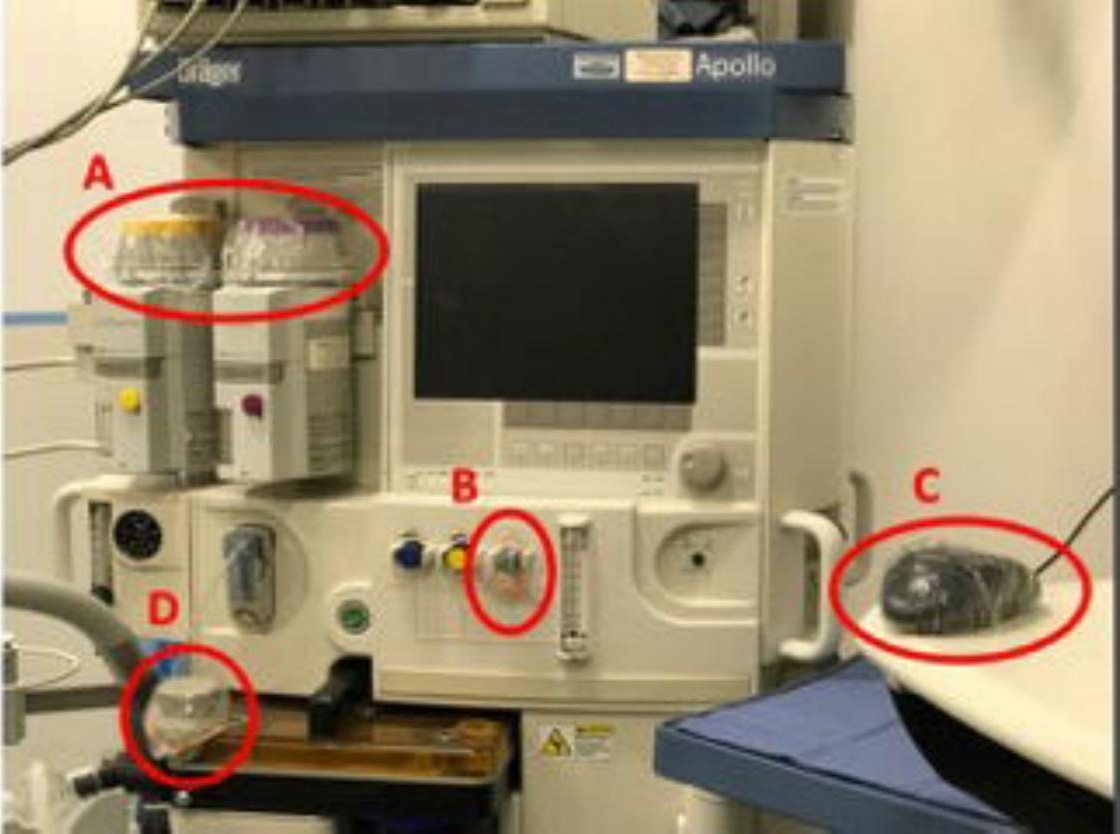



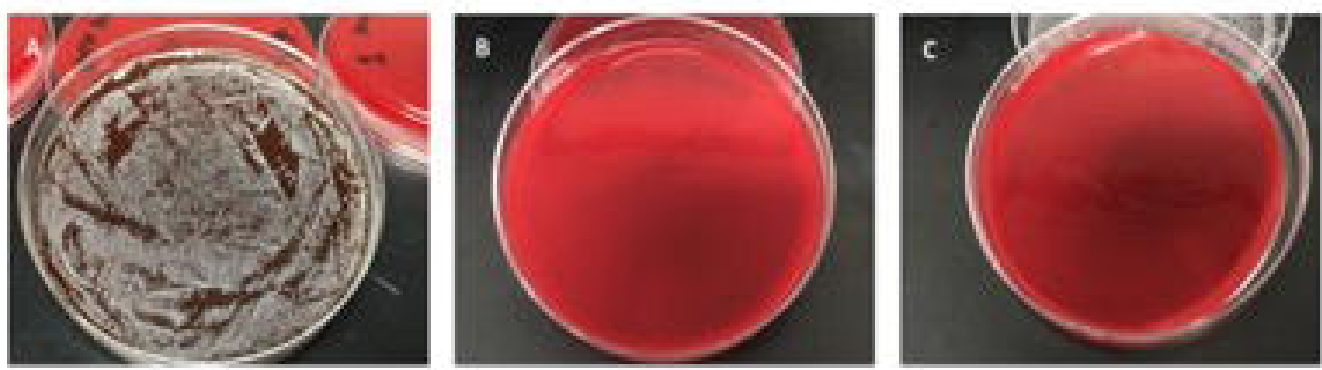


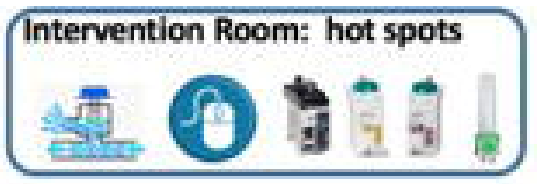

\section{Baseline culturing}

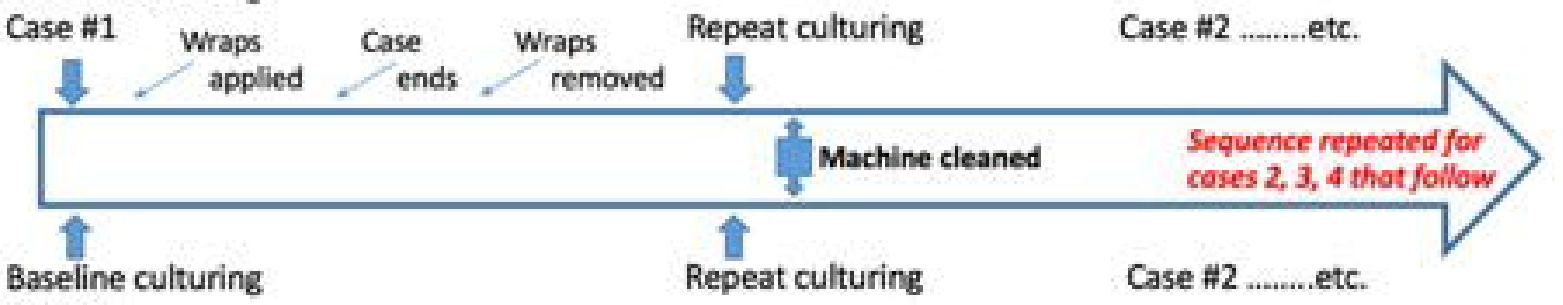

\section{Case H1}

\section{Control Room: hot spots}

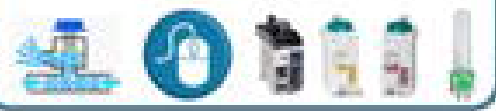

Legend: "hot spots" noted left to right: breathing circuit pressure control, mouse, 3 anesthetic vaporizers, $\mathrm{O}_{2}$ flowmeter control

Cases in each room were matched as closely as possible for complexity and duration

Institution has a dedicated, protocol driven anesthesia machine cleaning crew 